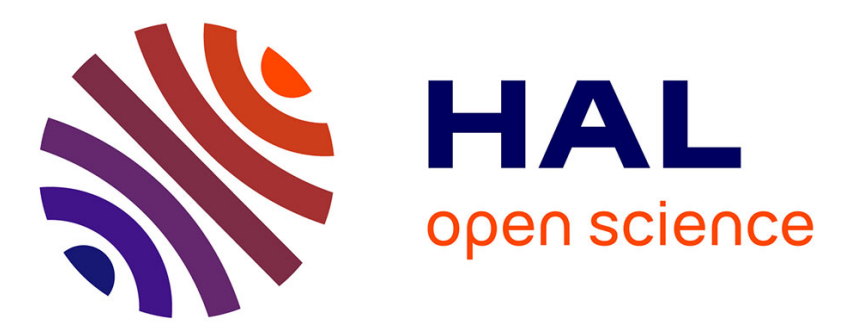

\title{
Towards an understanding of weak turbulence close to the convection threshold in large aspect ratio systems
}

P. Manneville

\section{To cite this version:}

P. Manneville. Towards an understanding of weak turbulence close to the convection threshold in large aspect ratio systems. Journal de Physique Lettres, 1983, 44 (22), pp.903-916. 10.1051/jphyslet:019830044022090300 . jpa-00232281

\section{HAL Id: jpa-00232281 https://hal.science/jpa-00232281}

Submitted on 1 Jan 1983

HAL is a multi-disciplinary open access archive for the deposit and dissemination of scientific research documents, whether they are published or not. The documents may come from teaching and research institutions in France or abroad, or from public or private research centers.
L'archive ouverte pluridisciplinaire HAL, est destinée au dépôt et à la diffusion de documents scientifiques de niveau recherche, publiés ou non, émanant des établissements d'enseignement et de recherche français ou étrangers, des laboratoires publics ou privés. 
Classification

Physics Abstracts

$47.25 \mathrm{~J}-47.25 \mathrm{M}$

\title{
Towards an understanding of weak turbulence close to the convection threshold in large aspect ratio systems
}

\author{
P. Manneville \\ DPh-G/PSRM. Orme des Merisiers, CEN-Saclay, 91191 Gif sur Yvette Cedex, France
}

(Reçu le 13 juillet 1983, accepté le 3 octobre 1983)

\begin{abstract}
Résumé. - Nous analysons l'évolution d'un transitoire turbulent observé dans une simulation numérique en géométrie rectangulaire à nombre de Prandtl modéré sur un modèle simplifié de convection incluant les effets des écoulements de dérive. Un processus particulier de convergence de ces écoulements, compression des rouleaux et nucléation de dislocation apparaît jouer un rôle répété. Le comportement non-relaxationnel observé semble résulter d'une "frustration dynamique " due a un défaut de compatibilité entre les écoulements à grande échelle et la courbure générale de la structure pouvant « expliquer » l'existence de la turbulence faible en géométrie étendue.
\end{abstract}

\begin{abstract}
We analyse the evolution of a turbulent transient observed in a numerical simulation in rectangular geometry at moderate Prandtl number on a simplified model of convection which includes the effects of drift flows. A particular process of drift flow convergence, compression of the rolls and dislocation nucleation is seen to play a repeated rôle. The non-relaxational behaviour observed seems to follow from a «dynamical frustration " due to a lack of compatibility between the drift flow and the overall curvature of the pattern, which could " explain " the occurrence of weak turbulence in extended geometry.
\end{abstract}

\section{Introduction.}

Recent advances in the field of the transition to turbulence have led to distinguish the case of confined geometry where weak turbulence means basically temporal chaos from the case of extended geometry where, due to weaker lateral boundary effects, spatial inhomogeneities are expected to complicate the picture [1]. In particular it is not yet known if the evolution of natural textures should always lead to steady states or if truly turbulent states can exist close to the threshold in Rayleigh-Bénard convection. The final word would be given if one could derive a Lyapunov functional from which the most general evolution could follow close to the threshold. Unfortunately present restrictions to such a formulation [2] forbid any firm conclusion. The difficulty of this problem is due for a large part to the coexistence of several spatio-temporal scales with very different orders of magnitude. For convection between rigid plates it is now well established that the evolution is relaxational on spatio-temporal scales small enough for the Newell-Whitehead-Segel amplitude equations [3] to be valid at lowest order, i.e. over lengths and times scaled by the coherence length $\xi=\xi_{0} / \sqrt{\varepsilon}$ and the relaxation time $\tau=\tau_{0} / \varepsilon$ with $\varepsilon=\left(R-R_{\mathrm{c}}\right) / R_{\mathrm{c}}$. However this property already breaks down for stress free convection due to the generation of «drift flows " accounting for the presence of vertical vorticity [4]. Up to now 
it is not known how serious the effect of higher order corrections is in the rigid case but one can guess that those coming from drift flows will be the most dangerous [5, 6]. Drift flow effects were included neither in previous theories of textures [7] nor in numerical experiments [8, 9] using simplified models of convection $[10,11]$. On the other hand, in simulations of reference 12 using the full primitive equations the drift flow was taken into account fully but implicitly. Here we shall use a model which has been derived recently and which isolates their contribution explicitly [13].

\section{The model and the conditions of the simulation.}

Our model involves only the local amplitude $W$ of the first vertical harmonic of the vertical velocity component and the $z$-independent horizontal drift flow $\mathbf{V}_{\mathbf{h}}$. This model reads :

$$
\tau_{0} \partial_{t} W=\left[\varepsilon-\left(\xi_{0}^{2} / 4 q_{0}^{2}\right)\left(\Delta_{\mathrm{h}}+q_{0}^{2}\right)^{2}\right] W-g\left[\left(\nabla_{\mathrm{h}} W\right)^{2}+q_{0}^{2} W^{2}\right] W-\tau_{0} \mathbf{V}_{\mathrm{h}} \cdot \nabla_{\mathrm{h}} W
$$

where $\Delta_{\mathrm{h}}=\partial_{x x}^{2}+\partial_{y y}^{2}$ and $\nabla_{\mathrm{h}}=\left(\partial_{x}, \partial_{y}\right)$. The linear operator generates periodic structures with wave-lengths close to $\lambda_{0}=2 \pi / q_{0}$. The first harmonic of the temperature field and the corresponding periodic horizontal flow components are proportional to $W$ and $\nabla_{\mathrm{h}} W$, respectively. The cubic term is similar to that of previous simplified models $[10,11]$ except that it does not derive from a potential in agreement with what one can guess for actual convection. $\mathbf{V}_{\mathbf{h}}$ derives from a stream function through :

$$
\mathbf{V}_{\mathrm{h}}=\left(-\partial_{y} \phi, \partial_{x} \phi\right)
$$

which in turn is governed by :

$$
\left(\partial_{t}-P \Delta_{\mathrm{h}}\right) \Delta_{\mathrm{h}} \phi=\left(1 / q_{0}^{2}\right)\left(\partial_{x} W \Delta_{\mathrm{h}} \partial_{y} W-\partial_{y} W \Delta_{\mathrm{h}} \partial_{x} W\right)
$$

This equation is nothing else than that for the $z$-independent part of the vertical vorticity $\left(\zeta_{z}=\Delta_{h} \phi\right)$ and $P$ is the Prandtl number which weights the respective rôle of the thermal and hydrodynamic variables in convection. This model is (nearly) exact at the limit $\varepsilon \rightarrow 0$ for convection between stress free plates and allows to recover the Siggia-Zippelius modified amplitude equations [4] at lowest order. For rigid plates the left hand side of $(1 b)$ is slightly different [13].

To a certain extent, simulations with periodic boundary conditions (BC) [12] may be understood as corresponding to an infinite aspect ratio system with a cut-off at the longest wave-lengths but they are not adapted to a direct comparison with laboratory experiments in laterally finite containers. On the other hand one can try to study finite size and geometrical effects by adding to the model equations adequate $\mathrm{BC}$ at the border line of the horizontal domain under consideration. Here they read $[14,13]$ :

$$
W=\hat{\mathbf{n}} \cdot \nabla_{\mathrm{h}} W=\phi=\hat{\mathbf{n}} \cdot \nabla_{\mathrm{h}} \phi=0
$$

where $\hat{\mathbf{n}}$ is the unit vector normal to the boundary. They express the fact that all velocity components must vanish at the lateral walls.

Here we present results of simulations performed in a rectangular domain of length 50 and width 36 , the length unit being such that $q_{0}=1$ instead of $\pi / \sqrt{2}$ as for stress free convection (several other convenient changes of scale have been performed in equations 1 in order to minimize the number of products by physically irrelevant constant factors in the numerical application). Relative to the width of a cell $\left(\lambda_{0} / 2=\pi\right)$ these dimensions correspond to aspect ratios $\Gamma_{x}=50 / \pi=15.9$ and $\Gamma_{y}=36 / \pi=11.5$, thus the box is sufficiently large to expect effects typical of large aspect ratios but sufficiently small to reach the asymptotic regime within a reasonable period of time.

We have developed a finite difference code which is $\mathcal{O}\left(h^{2}\right)$ and $\mathcal{O}(\delta t)$ where $h=\delta x=\delta y$ is the space step and $\delta t$ the time step. In order to avoid stringent numerical stability conditions [15] the 
linear operators are treated implicitly while the nonlinear terms remain explicit. The resulting block penta-diagonal matrices are solved using a standard block elimination technique [16]. The final algorithm is numerically stable in such a way that allows us to choose the time step $\delta t$ not from stability requirements but from accuracy considerations. A reasonable compromise between efficiency and reliability has been found for $h=1\left(6 \mathrm{pts}\right.$. $\left./ \lambda_{0}\right)$ and $\delta t=0.25$ during " rapidly varying " phases but much larger when the evolution was «slow ». A comparison with steady states obtained after halving $h$ and with unsteady regimes after doubling $\delta t$ gives us confidence in our results.

\section{Preliminaries.}

We have performed a rather systematic exploration of the plane of the control parameters $\varepsilon$ and $P$ in the domain $0 \leqslant \varepsilon \leqslant 0.5$ and $0 \leqslant 1 / P \leqslant 1$. Initial conditions were either a low level random noise ( $\mathrm{R}$-type experiment) or a previous final state (C-type, $\mathrm{C}$ for continuation). In agreement with previous observations, for R-type simulations and $\varepsilon$ small enough (say $\varepsilon=0.1$ ) we have obtained regular structures made of rolls parallel to the short side of the cell. For larger $\varepsilon$, typically 0.5 , at large $P$ we have obtained textures analogous to those already found using simplified model appropriate to $P=\infty$ (see Fig. 1 for $P=5$ ). But at small $P(=1)$ a more regular
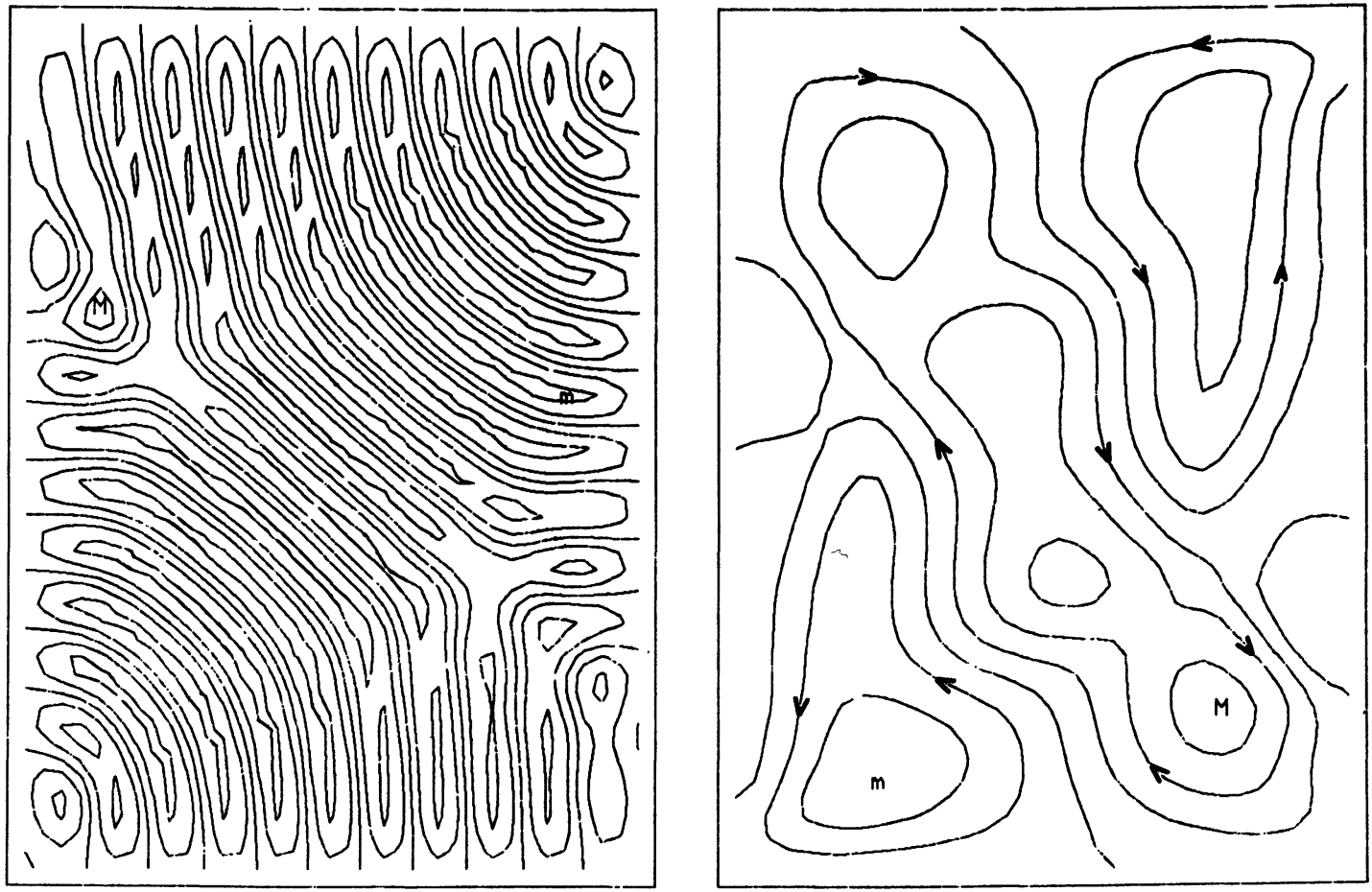

Fig. 1. - Steady state obtained at $\varepsilon=0.5$ and $P=5$ for low level noisy initial conditions (R-type experiment). Left : convective variable $W$. M denotes the (positive) maximum of $W(x, y)$ and $\mathrm{m}$ the (negative) minimum. Let $\mu=\max (\mathrm{M},-\mathrm{m})$ be the maximum of $|W|$ over the cell. We draw the 5 level curves $W(x, y)=2 \mu / 3, \mu / 3,0,-\mu / 3$ and $-2 \mu / 3$. Right : stream lines of the drift flow (lines of constant $\phi$ ). From the definition one deduces that the flow runs clockwise around a relative maximum and counterclockwise around a relative minimum as indicated by the arrow heads. 
steady structure has been found (Fig. 2) with slightly bent rolls nearly parallel to the long side of the cell and an associated drift flow in equilibrium $\left({ }^{1}\right)$ with the curvature of the rolls. In order to check the rôle of the drift flow in explaining the differences between large and low $P$ we have performed a series of $C$-type experiments using textures obtained at large $P$ as initial conditions for simulations at lower $P$. This procedure seems to be difficult to achieve in laboratory experiments but is not unconceivable since one can make $P$ vary without changing the fluid by simply choosing adequately the average temperature using liquid helium [16] or water [17] for example. The first experiment $(\varepsilon=0.5$ and $P=1$ starting from $P=\infty$ ) has led to straight rolls parallel to the short side of the cell after a (relatively) short transient. Here we report on another much less brutal experiment which turns out to be more interesting.
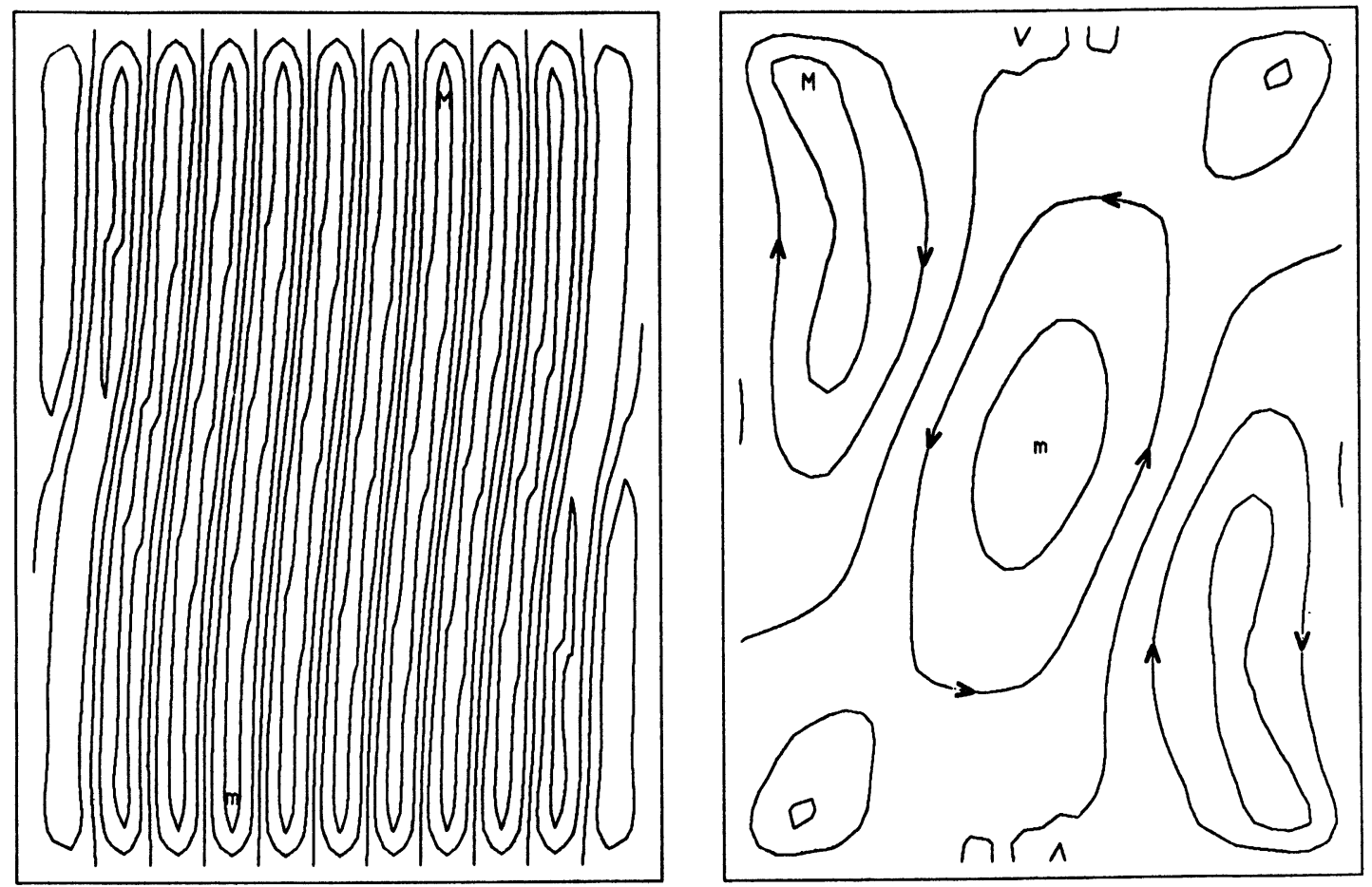

Fig. 2. - Steady state obtained at $\varepsilon=0.5$ and $\operatorname{Pr}=1$ (R-type exp.).

Starting with the texture obtained at $\varepsilon=0.5$ and $P=5$ (Fig. 1) we have decreased $P$ progressively by steps, waiting a sufficient time for a steady state to settle down, then multiplying $P$ by $4 / 5$, etc... From $P=5$ to $P=3.2$ we observe only slight adjustments and correspondingly a relaxational behaviour on a rather short time scale. For $P=2$ the system tries to escape from the attraction basin of the current texture, but after a trial it comes back to it. As can be seen in figure 3, from a topological point of view the situation is the same as for $P=5$ (Fig. 1). The most interesting things happen when $P$ is further decreased to 1.6 in which case the structure is seen to suffer a reorganization that we now present in detail.

( $\left.{ }^{1}\right)$ Using Siggia-Zippelius modified amplitude equations [4] one can show that the direction of the drift flow is such that it tends to decrease the curvature. In a finite box we say that the drift flow is in equilibrium with the curvature of the rolls if, this flow is roughly perpendicular to the rolls and in the right direction wherever the curvature is large, the flow lines closing roughly parallel to the rolls in the inflexion region separating two domains with opposite curvatures. 

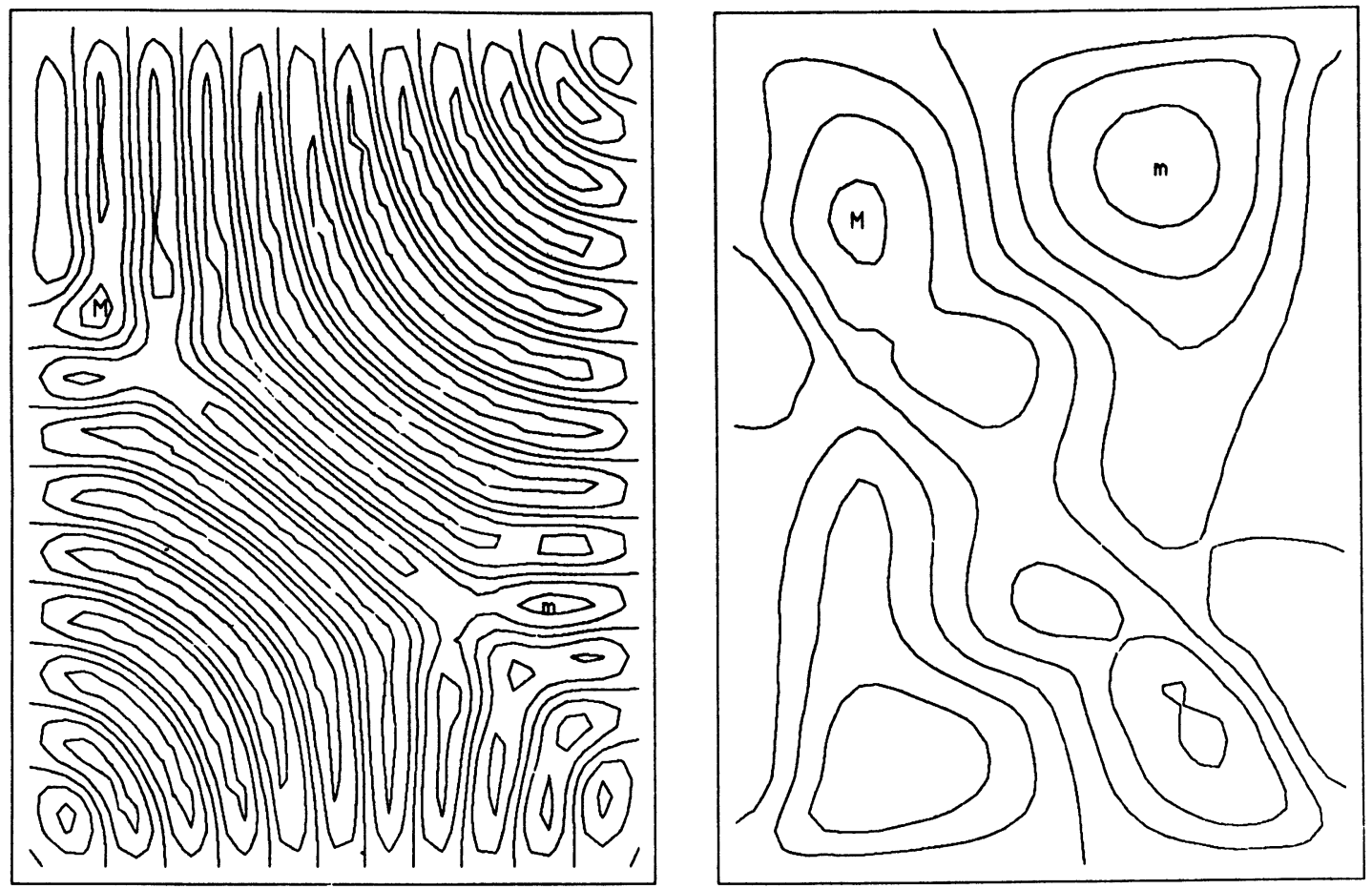

Fig. 3. - Steady state obtained at $\varepsilon=0.5$ and $P r=2$ (C-type exp.). Initial state for the evolution depicted in the following figures.

\section{An interesting turbulent transient at $P=1.6$.}

The film of the evolution is given in figures 5 to 13 . In addition figure 4 displays the variations of two global quantities deduced from the numerical solution : the convective heat flux and the amount of kinetic energy stored in the large scale drift flow. The convective heat flux $H$ is given by $H=\langle w \theta\rangle$ where $\langle\ldots\rangle$ means the horizontal average. To the order at which we work $\theta$ is simply proportional to $w$ and we have :

$$
H \propto \int_{s} W(x, y) \mathrm{d} x \mathrm{~d} y .
$$

In the same way the average kinetic energy $K$ reads $K=\left\langle V_{h}^{2} / 2\right\rangle$ or :

$$
K \propto \int_{s}\left(\nabla_{\mathrm{h}} \phi\right)^{2} \mathrm{~d} x \mathrm{~d} x .
$$

In figure 4, units for $H$ and $K$ are somewhat arbitrary but relative variations are meaningful and it will be seen that all the accidents which appear on these curves can be interpreted in terms of local events in the structure.

Starting with the final state for $P=2$ depicted in figure 3 we set $P=1.6$. The first phase denoted as " init " in figure 4 is a relaxation of the kinetic energy towards a value which is $(5 / 4)^{2}$ times the final value at $P=2$. Since the structure and thus the gradients of $W$ have not yet had time to change, this corresponds to the response of $\phi$ to a sudden increase of the right hand side of equa- 


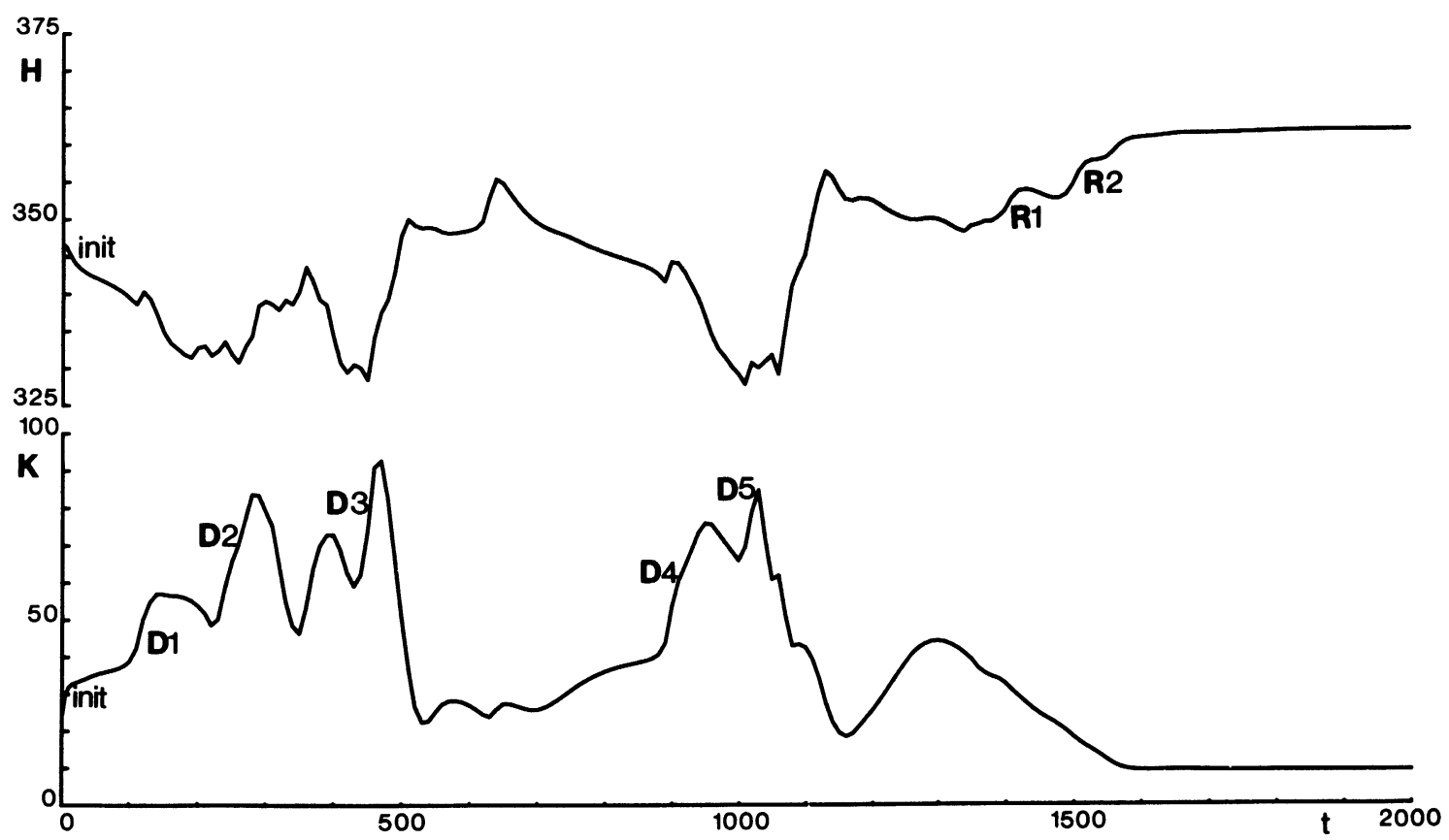

Fig. 4. - Variation of the convective heat flux ( $H$, top) and the kinetic energy stored in the large scale flow $(K$, bottom) during the turbulent transient. The meaning of the different labels is explained in the text.
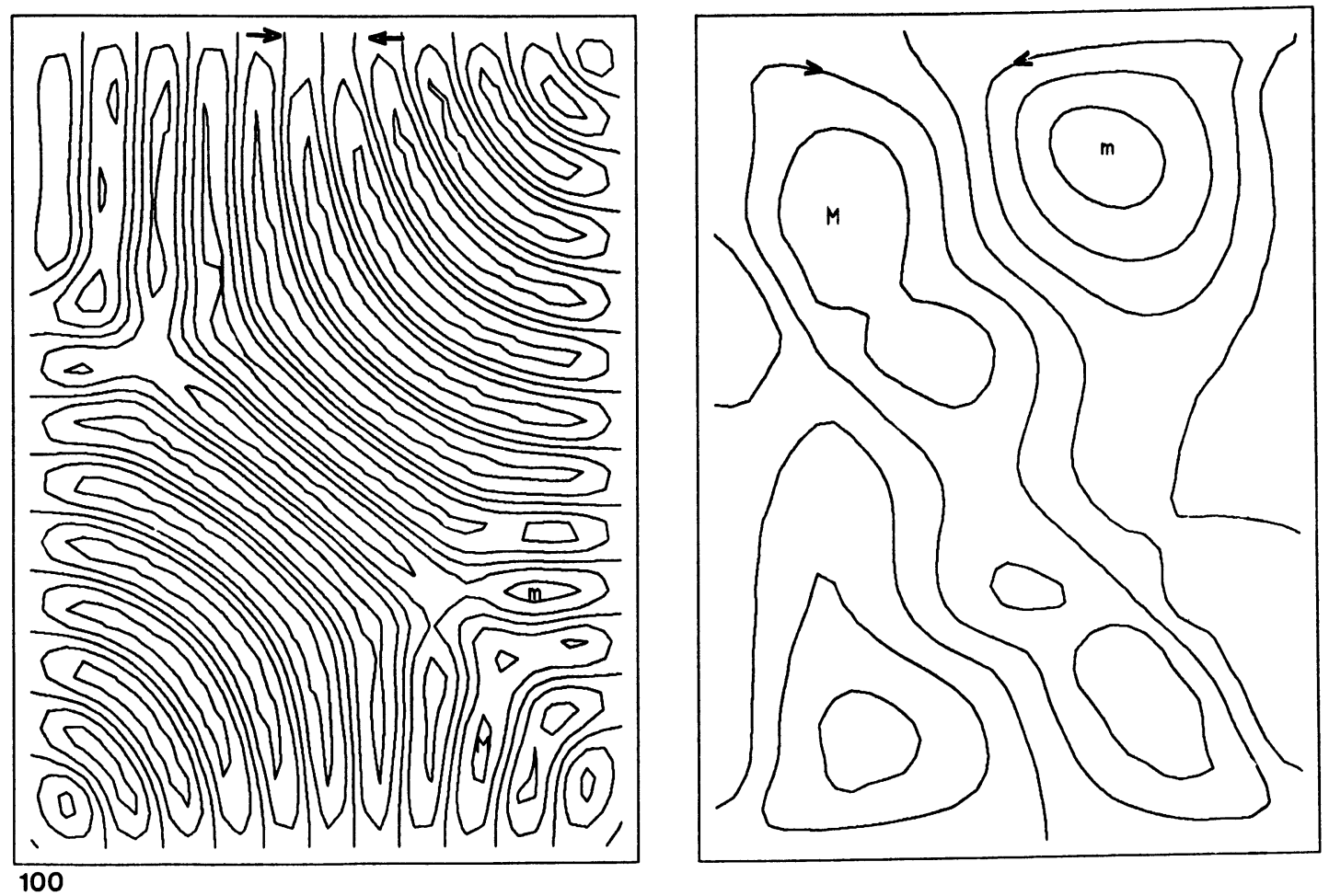

Fig. 5. - Situation at $t=100$. Explanations are given in the text. 

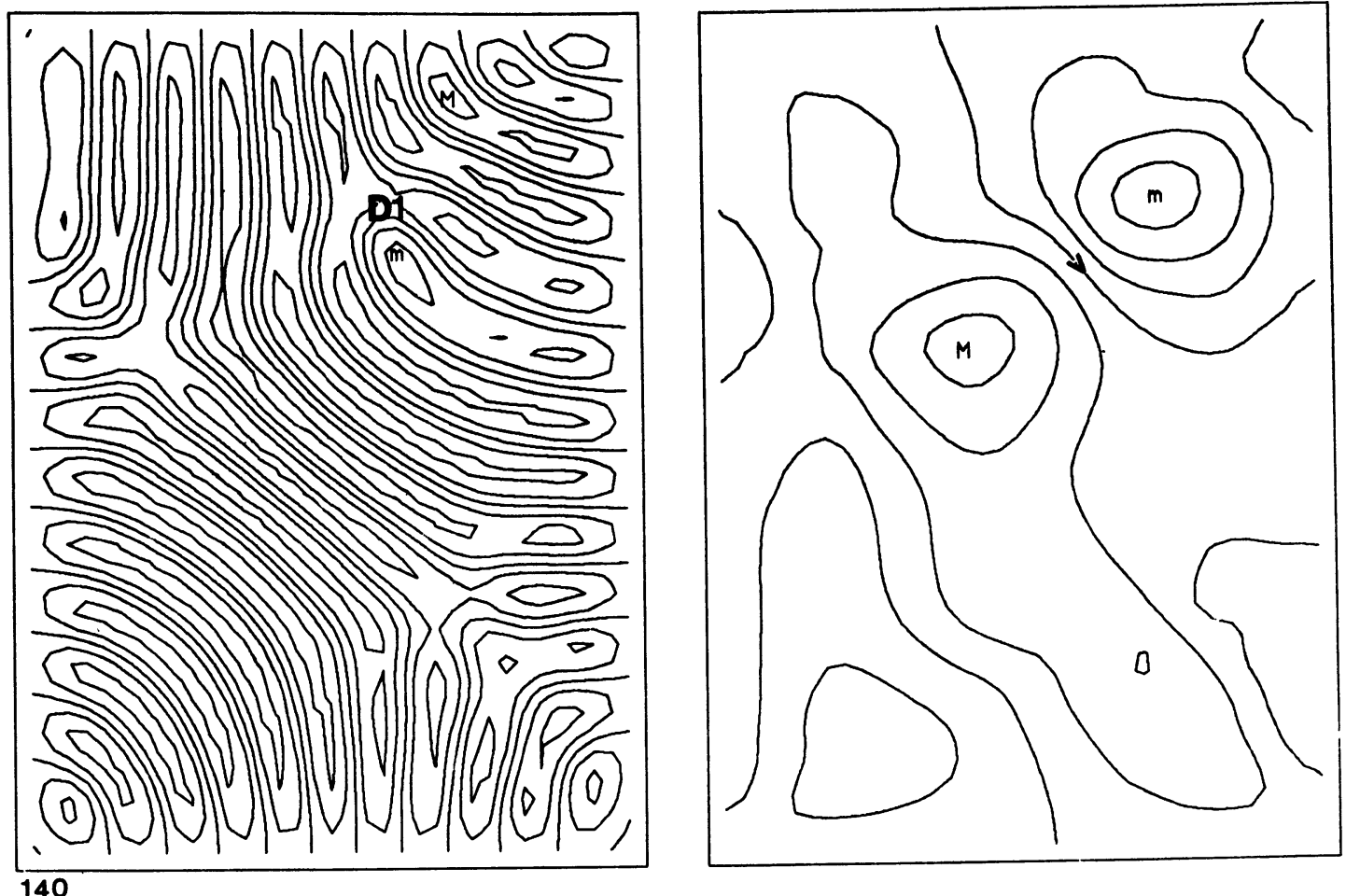

Fig. 6.
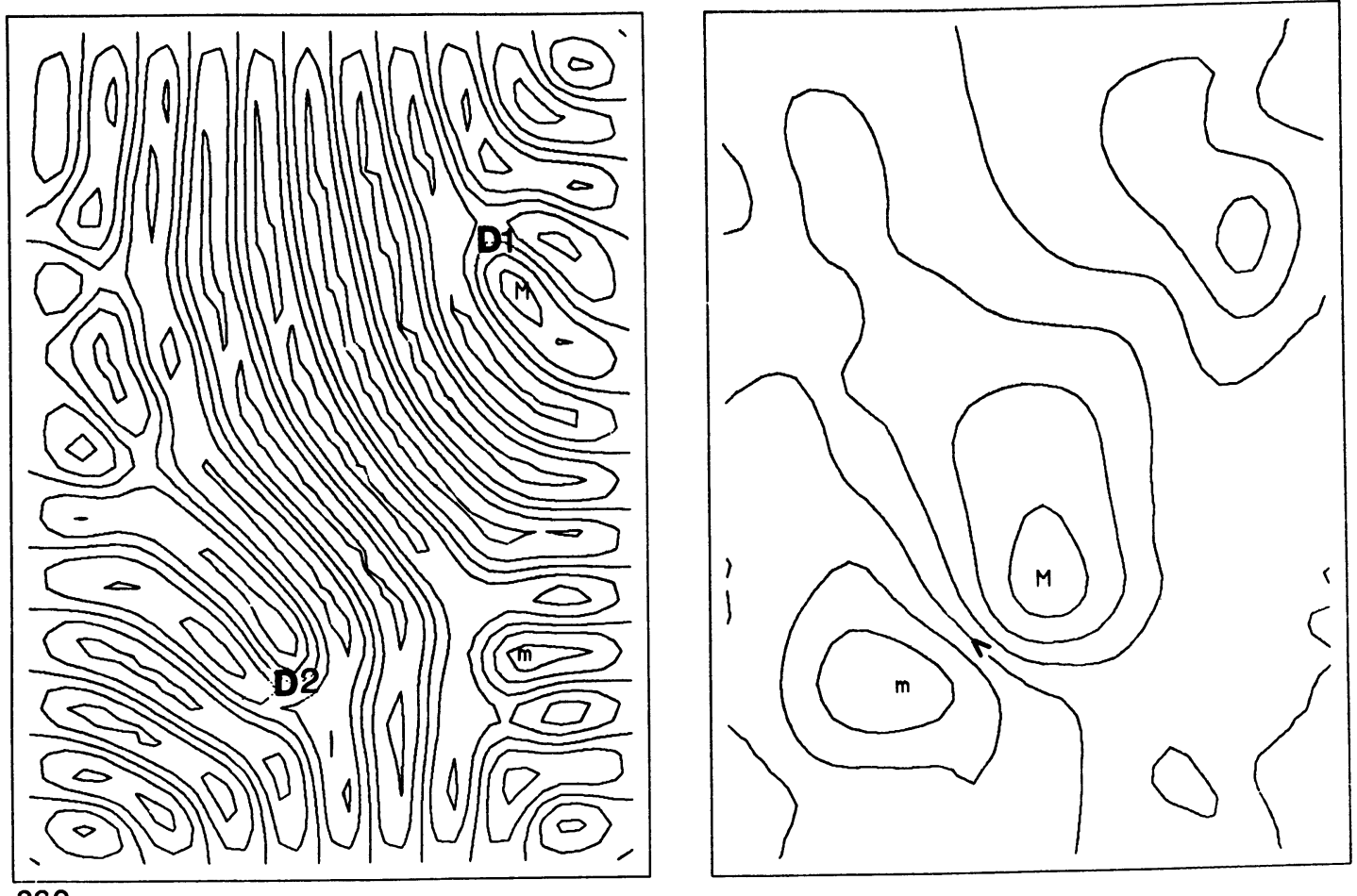

Fig. 7. 
tion $1 b$ by a factor $5 / 4$. The end of this relaxation process at about $t=70$ marks the actual beginning of the experiment.

What occurs then is not a slight adjustment of the structure to the new intensity of the drift flow as observed before but a much more dramatic evolution. In the upper part of figure 5 at $t=100$ we see that the convergence of the drift flow (already noticeable before, see Fig. 3 ) induces a compression of the central roll close to the boundary (there is also a less visible but measurable dilation of the extreme left roll). The corresponding stress is relaxed through the nucleation of a dislocation D1 which further climbs inside the cell $(t=140$, Fig. 6$)$ thus eliminating the supplementary pair of rolls. The secondary flow is seen to take a dipolar shape around the dislocation core and to rotate so as to sustain the motion of the dislocation [19]. All this corresponds to the first marked increase of kinetic energy in figure 4. Dislocation D1 then gets stuck to the wall on the right and the associated flow decreases slowly. A convergence analogous to that just described develops in the lower half inducing a second dislocation D2 which migrates from the lower side inwards. The resulting situation is depicted in figure 7 where we can see D1 and the new D2 with its associated flow sustaining the motion (see the 2nd increase of $K$ in Fig. 4).

The next phase is rather confused. A general cleaning in the upper left corner leaves a situation somewhat similar to the initial one. A dislocation nucleation, again linked to a convergence of the flow and a compression of the rolls, takes place at about $t=450$ (see the corresponding increase of $K$ ). This 3rd dislocation interacts with D1, which leads to some simplification in the upper right corner and one gets the pattern of figure 8 at $t=500$. A rather calm period begins, characterized by a relatively high heat flux and a comparatively small kinetic energy. Unfortunately it is easily checked that the secondary flow is not in equilibrium with the curvature of the texture in the centre : the curvature should induce a strong current from the left to the right in a region where the stream lines present a saddle point so that the corresponding flow nearly vanishes. The slow evolution which follows then corresponds to an attempt for better compatibility. This
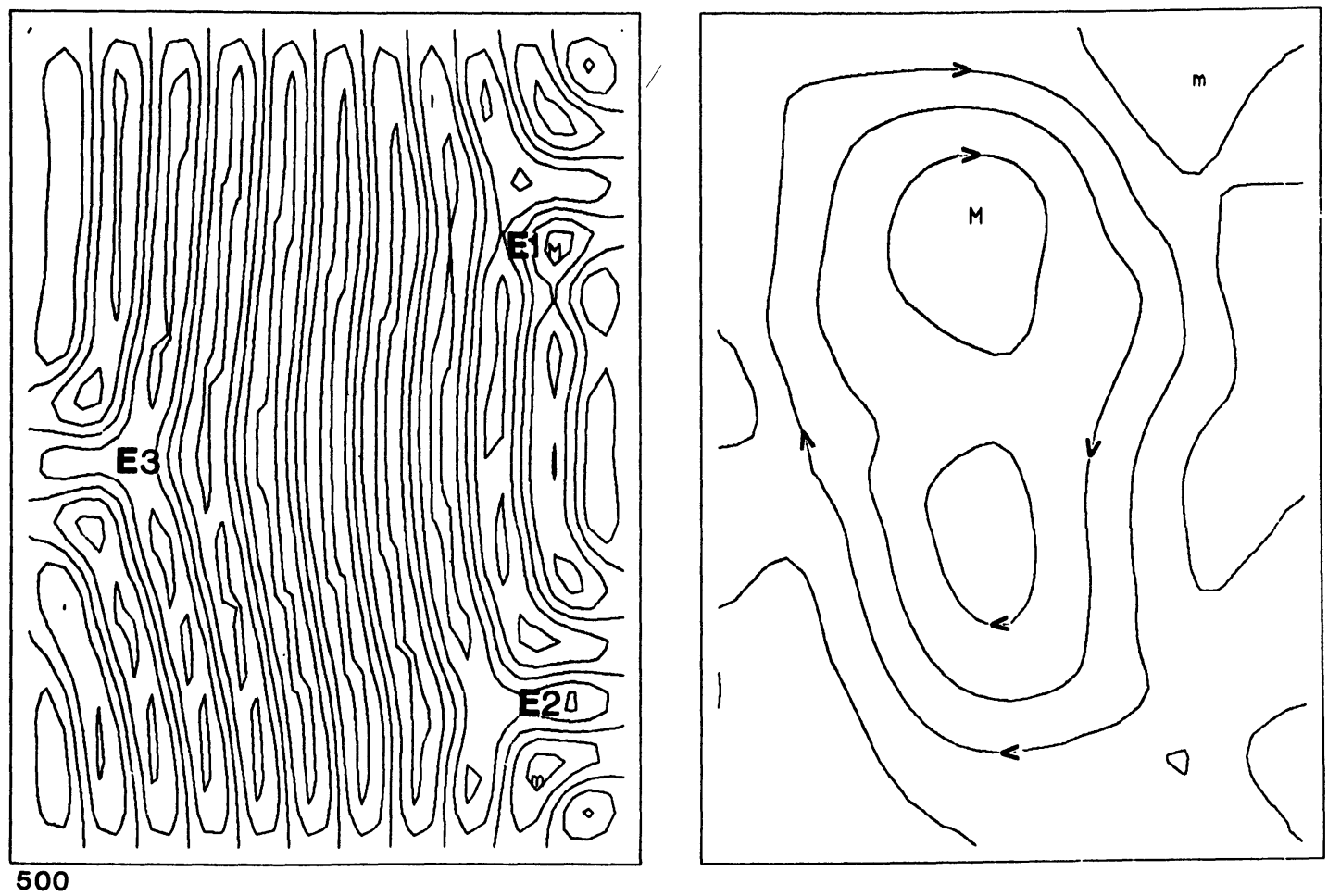

Fig. 8. 
ends in eliminating the defect E1 and in leaving a situation analogous to the initial one from the topological point of view $(t=700$, Fig. 9$)$ and moreover with comparable values of $H$ and $K$.
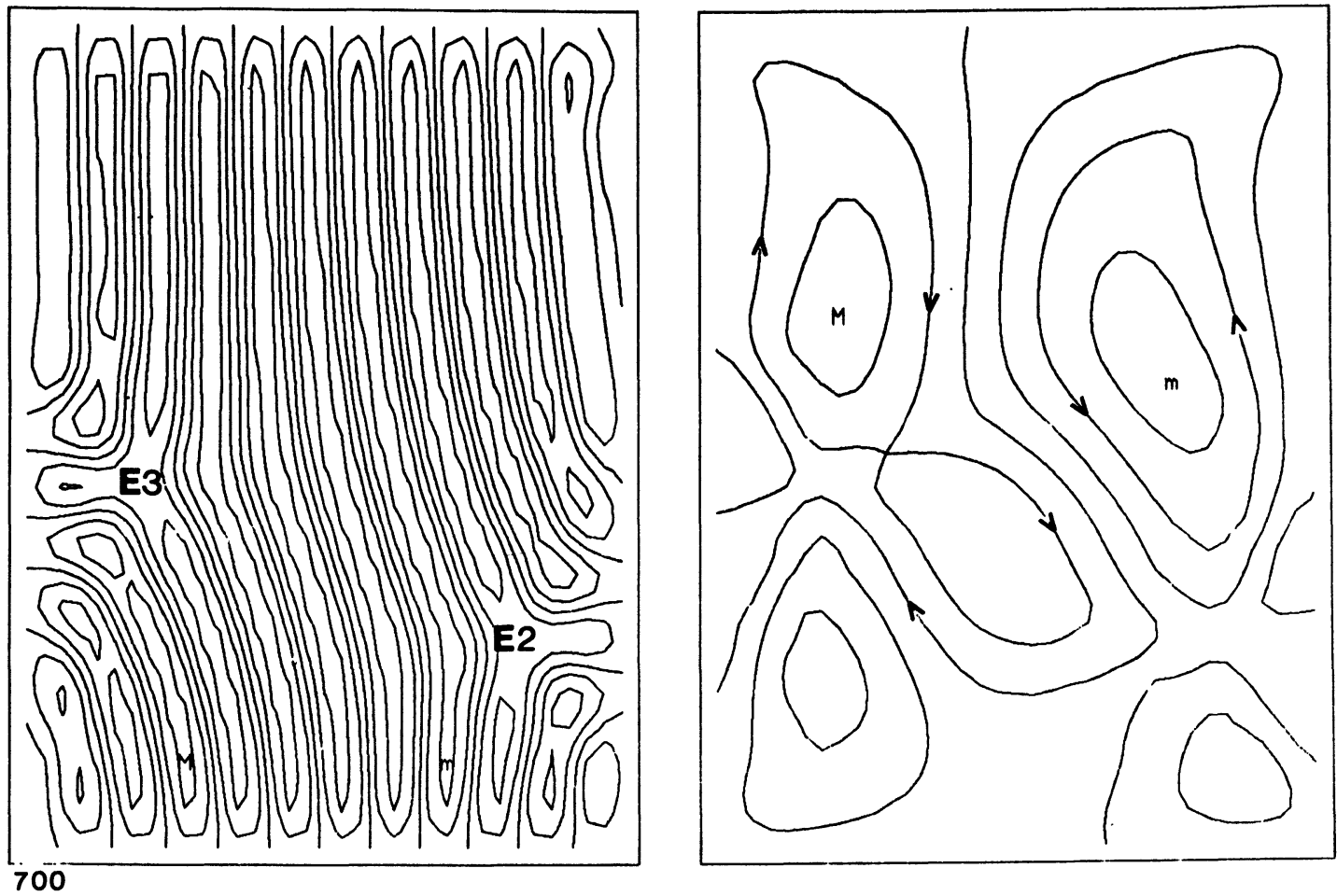

Fig. 9.

Same causes have the same effects : the process of convergence, compression, nucleation of dislocations repeats itself, dislocation D4 at $t \simeq 900$ and D5 at $t \simeq 1010$ in the upper half of the cell. At $t=1030$ we have still defect E3, D4 is blocked on the right side and D5 climbs in the structure (note again the aspect of the secondary flow and the increase of $K$ ). Then E3 disappears and some further cleaning takes place in the lower right corner so that we are left $(t=1080$, Fig. 11) with a single mixed defect $F$ (dislocation like in the centre and grain-boundary like close to the right side). The associated flow is not in equilibrium with the curvature of the rolls, which is easily checked by comparing figure 11 and figure $2:$ while the general aspects are similar, the flows run in opposite directions.

Contrary to what one could expect, the structure does not finds its way towards the attraction basin of the solution depicted in figure 2 and does not succeed in eliminating its defect completely. A slow rearrangement leads to the situation of figure 12 at $t=1400$. Defect $\mathrm{F}$ is partly « digested " and the flow becomes compatible with the roll curvature. The final phase is a slow relaxation towards a completely symmetrical pattern with a disclination on one side. During this last phase the trace of the elimination of the small rolls R1 and R2 in the corners of figure 12 can be found in the oscillations of the heat flux (Fig. 4) :R1 disappears at $t \simeq 1500$ and $\mathrm{R} 2$ at $t \simeq 1550$.

The final state is steady and corresponds to the largest convective heat flux and the smallest kinetic energy ever reached in the course of this evolution. In this context it should be noted that the heat flux is large as long as the structure is rather regular but decreases as soon as defects appear. This can be easily understood since defects involve regions where the convective variable 

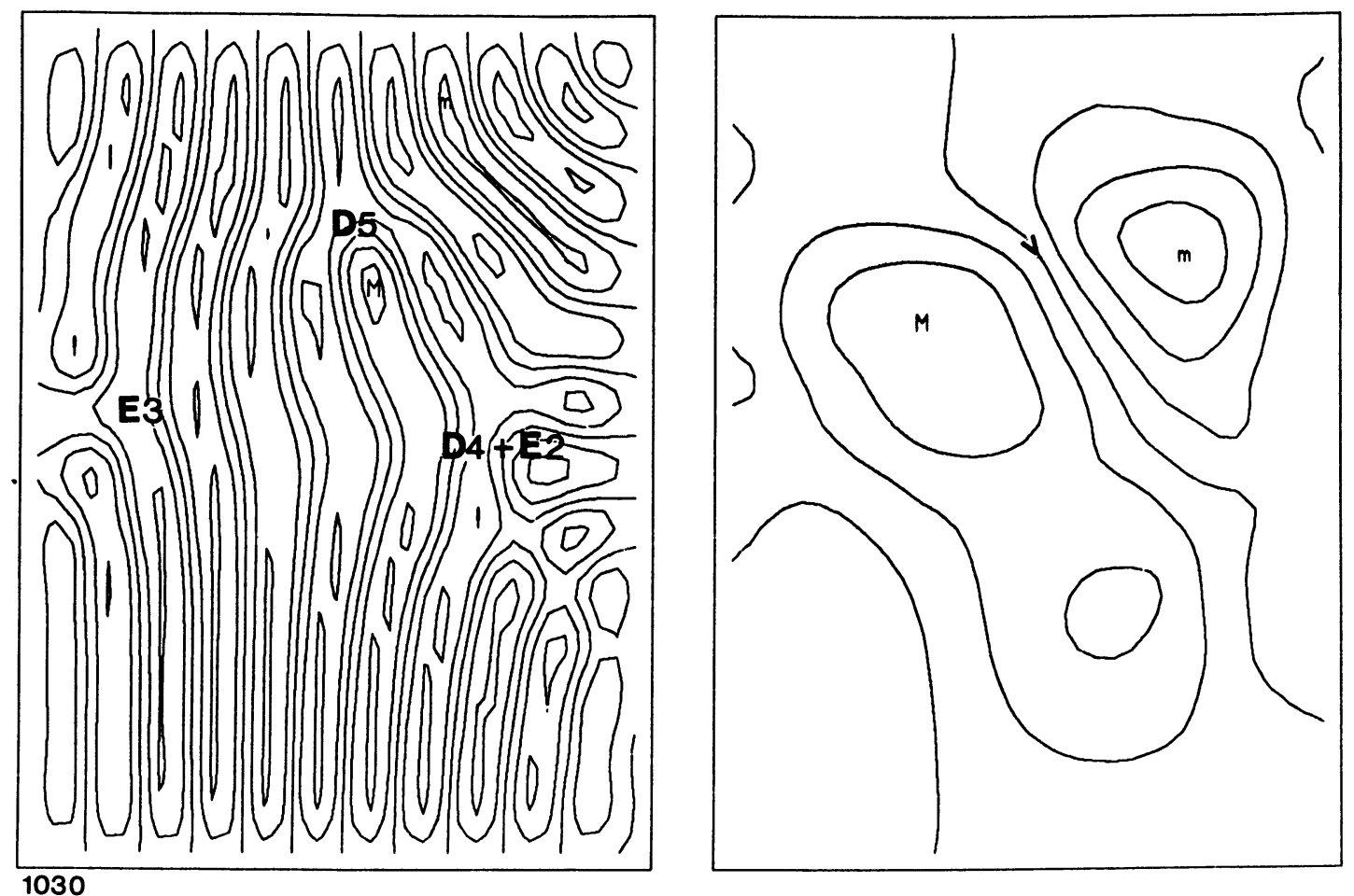

Fig. 10.
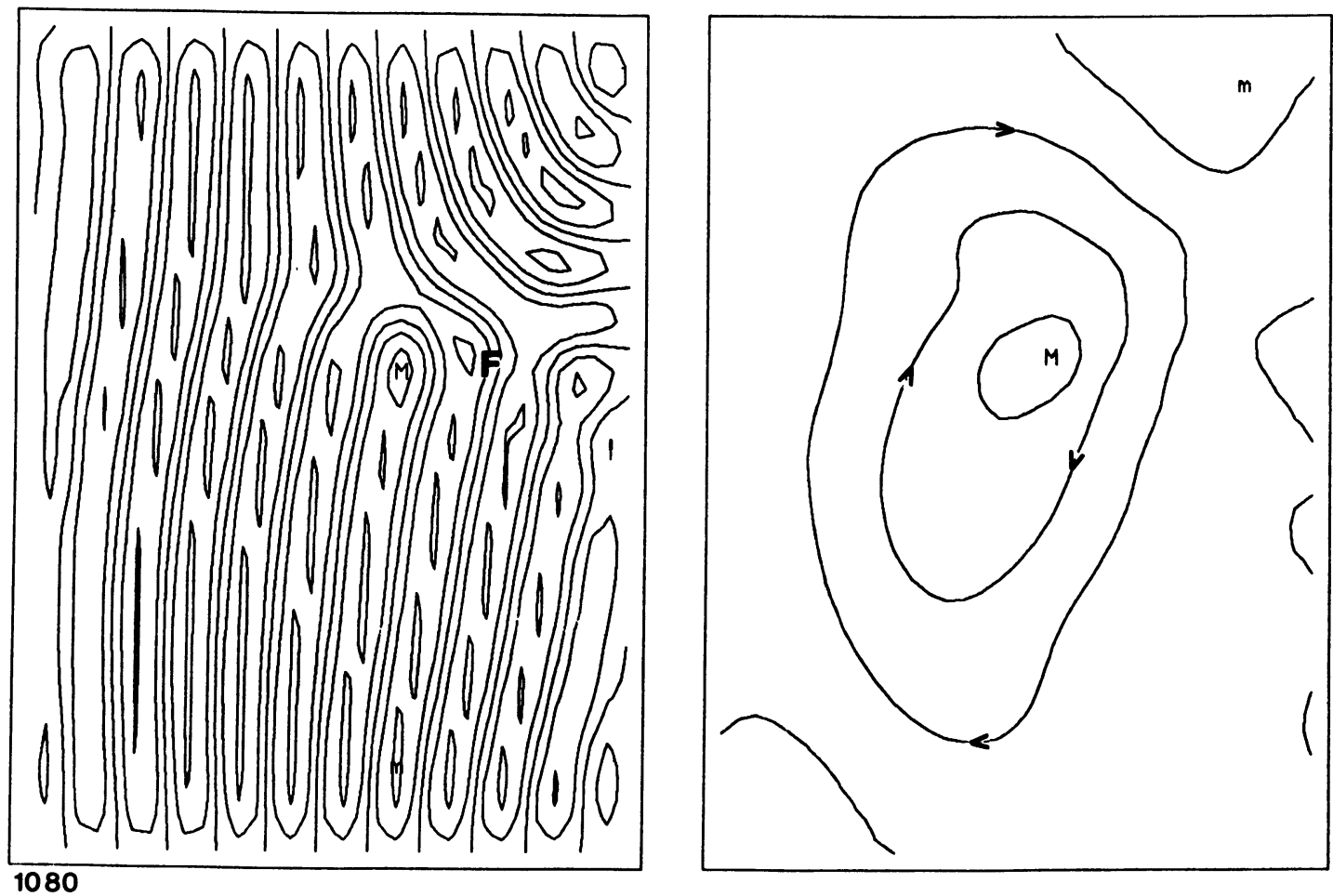

Fig. 11. 

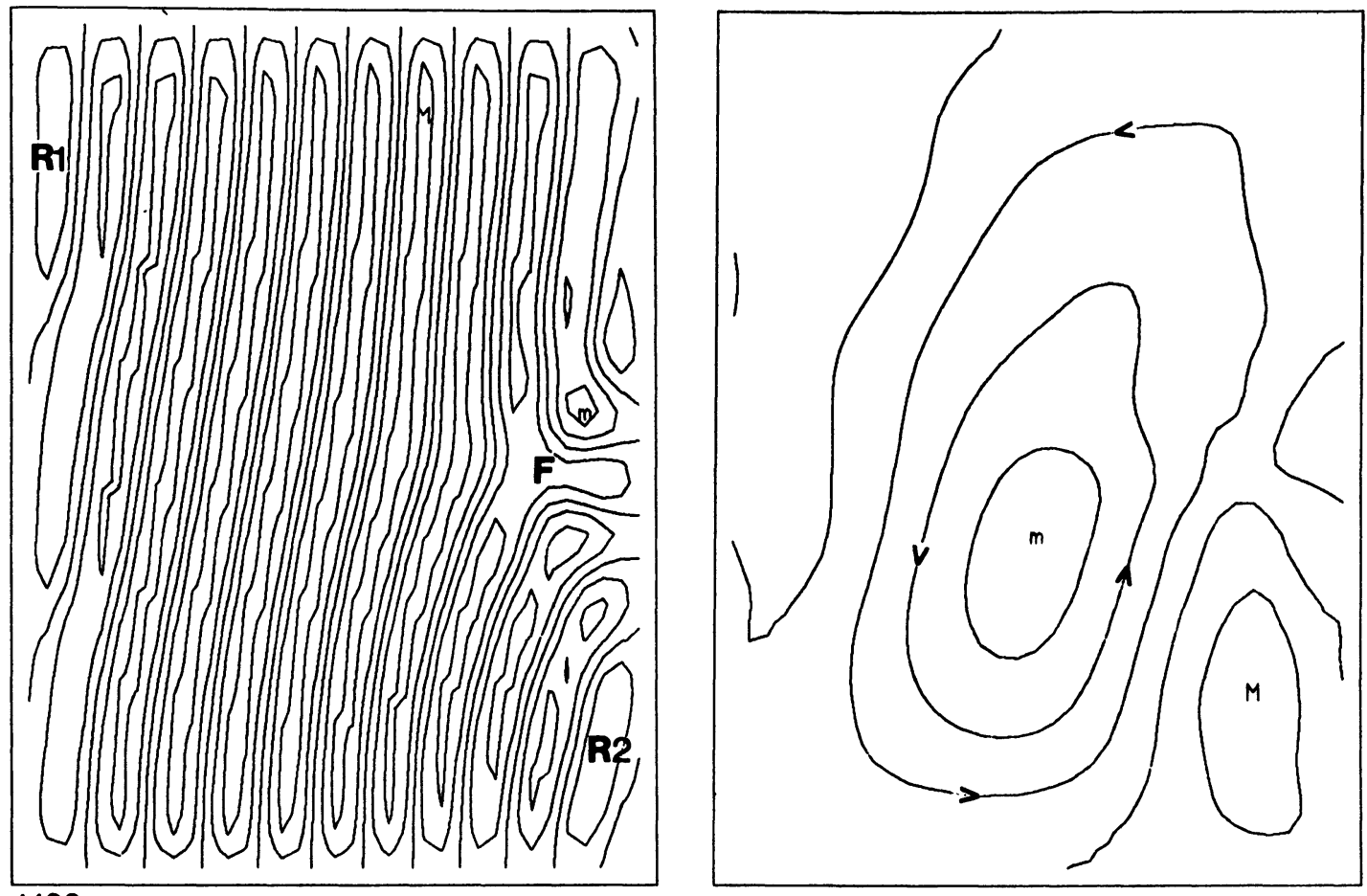

1400

Fig. 12.

$W$ becomes smaller on rather large regions. On the other hand the kinetic energy stored in the large scale flow increases with the number of moving dislocations and falls down as soon as they stop. Therefore calm periods are associated with large convective heat flux and small kinetic energy.

\section{Discussion - conclusion.}

At this point several concluding remarks may be in order. First, in spite of the comparatively small size of the cell, we have observed phenomena strikingly similar to those found in laboratory experiments, either from the point of view of the textures obtained (compare our Fig. 3 with Fig. $2 a$ of [18]) or from the point of view of the evolution during a transient (Fig. 3 of [18]), especially the succession of " active " and " calm " periods [20]. Another point to be mentioned is the observation of modulations of the " skewed varicose " (SV) type [21]. These deformation modes are thought to play an important rôle in the transition to chaotic time dependence at moderate Prandtl numbers and large aspect ratios [18]. Here SV perturbations are visible in figures 8, 11, 12 and to a lesser extent in figures 2 and 13. Further work is necessary to prove that they correspond exactly to SV modes since a corresponding modulation of the drift flow would be expected but does not seem to be visible on stream charts. However this modulation can be small and completely overshadowed by the more intense general flow generated by curvature. In this connection let us point out that one often speaks of a threshold for SV instability but that this makes an implicit reference to a basic structure of perfect parallel rolls : this threshold is associated with a symmetry breaking. On the other hand, for already distorted rolls like those present in a given texture, the symmetry is broken from the start and the notion of a threshold disappears, i.e. we are in presence of an imperfect bifurcation and we observe the related, possibly very large, non- 

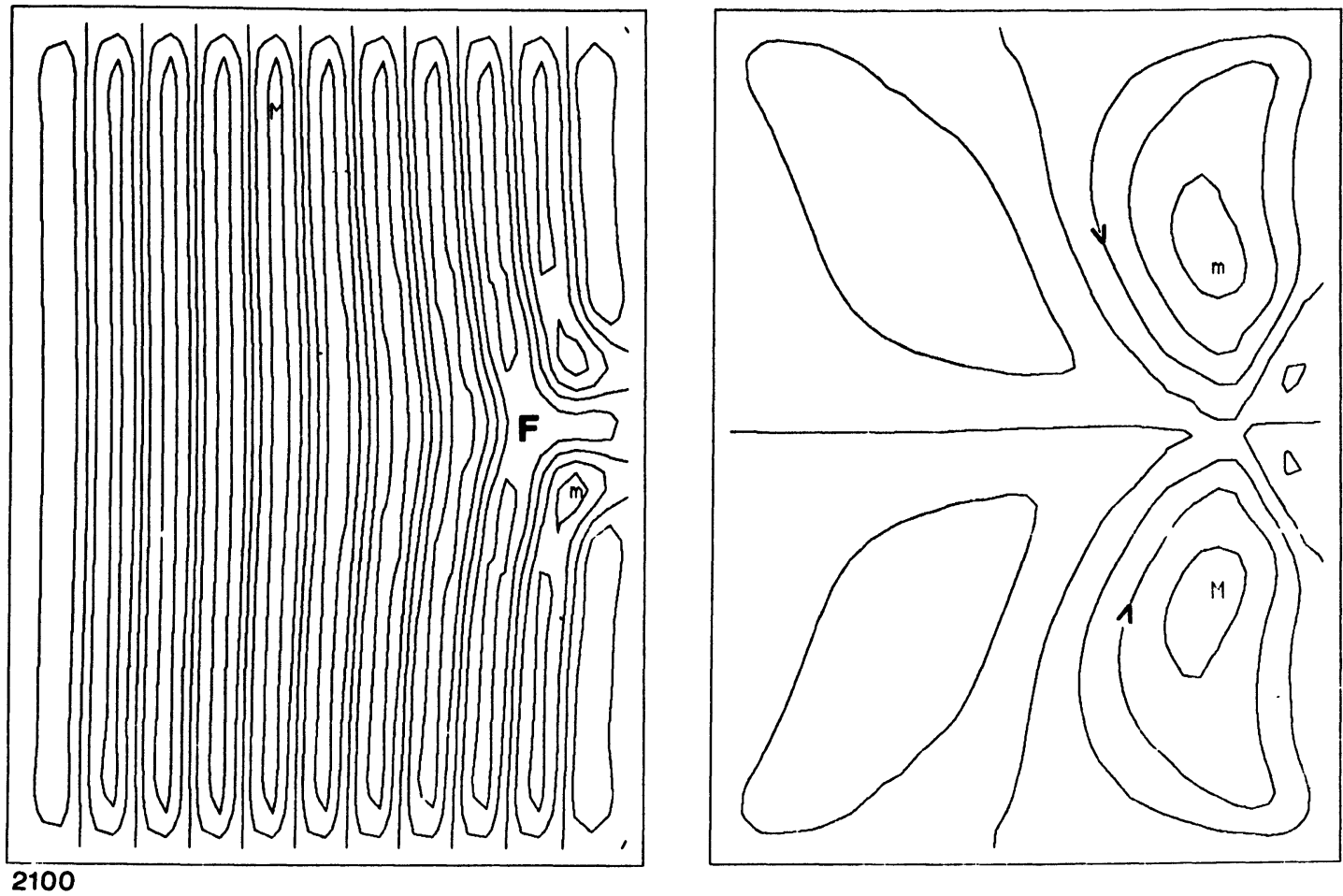

Fig. 13.

linear response to an external constraint. This may explain the transient SV-type modulations observed.

A thorough interpretation of laboratory experiments in terms of observations based on this model comes up against shortcomings inherent in any simulation on models. These shortcomings are twofold : numerical and physical. First it is quite clear that the numerical procedure can be improved by increasing the spatio-temporal resolution and using more sophisticated codes [8b]. The consideration of larger cells would also be welcomed. But all this is not very crucial. The limitations of physical origin linked to the appropriateness of the model are more fundamental. Their consequences are much more delicate to assess.

First of all, the use of free BC enhances the rôle of drift terms, introducing them at order $\varepsilon^{3 / 2}$ instead of $\varepsilon^{2}$ for rigid BC. In recent works on the subject, the recourse to free BC has been considered as having an appreciable heuristic value in suggesting reasonable phenomenological extensions to the case of rigid $\mathrm{BC}[4,12,5]$, quantitative comparisons being available for very special problems only [6]. From our simulations it seems that the over-estimation of the strength of the drift flow specific to free BC leads quite systematically to an excessive decrease of the amount of curvature of the rolls at small $P$ (several other trials with different initial conditions - not reported here - have led to straight rolls parallel to either the short or the long side of the cell). Such a trend may be less marked if drift flows are weaker, as is the case for rigid BC.

A second limitation, specific to the model, comes from the drastic truncation of the (vertical) modal expansion which is accurate at the limit $\varepsilon \rightarrow 0$ and for slowly varying modulations only. This is the price to be paid for singling out the interaction of the two most relevant fields close to the threshold. The corresponding poor vertical resolution results in uncontrolled perturbations, the nature of which is basically that of " aliasing errors " in ab initio numerical simulations. These perturbations will be greater far from the threshold and in strongly modulated regions. However 
the general aspect of the large scale flow, with only weak small scale anomalies, suggests that the truncation is reliable and that equation $1 b$ extracts most of the relevant effects linked to the curvature of the rolls for sets $(\varepsilon, P)$ considered up to now.

Let us now come to the positive aspects of our study. It does not seem necessary to quote again the great similarity between laboratory experiments and our findings but let us stress the definitely non-relaxational behaviour observed here. As a matter of fact it is difficult to understand the observed evolution as monotonous, even in the widely broadened sense which is implicit when one speaks of relaxation (think of a Lyapunov functional). The repetition of similar sequences and the succession of calm and active phases unavoidably evokes the notion of intermittency as first introduced in the theory of the transition to turbulence in small aspect ratio systems [22]. The novel point is that our simulation suggests a well defined physical origin for this behaviour when observed at large aspect ratios :

Assuming a given texture, it would be exceptional that the drift flow be in equilibrium with the curvature of the rolls. Compatibility would be achieved through a slow global reorganization of the pattern via an advection of entire parts of the roll structure. This process would imply a progressive concentration of local stresses on the rolls (compression, dilation or shear) ending at the nucleation of defects (dislocations). The motion of these just created defects would induce curvature modifications to which the drift flow should have to accomodate itself. This ever restarted search for equilibrium suggests the notion of a « frustration » of dynamical origin $\left({ }^{2}\right)$.

Beyond the particular mechanism which has acted repeatedly and which may be worth studying theoretically, this novel notion of frustration of dynamical origin combined with the older one linked to geometry could afford an explanation to the occurrence of turbulence close to the threshold [17]. In our simulations we have observed turbulent transients only. Of course this may be due to the small size of the box but, as already quoted, also to the over-estimation of the strength of the drift flow which topologically favours simple structures made of straight rolls. Quite paradoxically, in the realistic case, a weaker drift flow could allow for sustained weak turbulence in leaving the possibility of more complicated textures which would restore the compatibility problem between roll curvature and vertical vorticity.

Finally the recurrence of some typical but unstable textures at several steps of the evolution leads one to think of an instability of trajectories and a sensitivity to initial conditions in some very complicated phase space. Thus for large aspect ratios we would arrive at a picture strongly reminiscent of that valid for systems with few degrees of freedom [1].

\section{Acknowledgments.}

Part of this work has been developed thanks to a Cray CPU time allocation within the framework of the CRAY-CISI ATP of the CNRS. These results have been presented for the first time at the "Colloque sur les structures cellulaires dans les instabilités " organized at Gif-sur-Yvette (20-22 June 1983) by J. E. Wesfreid and S. Zaleski who should be thanked for their invitation. This letter has greatly benefited by discussions with P. Bergé and his group and with P. C. Hohenberg to whom the author is also indebted for a communication of Bell labs. preprints, references 2 and $8 b$.

$\left({ }^{2}\right)$ Remark that this description implies that the short term evolution of defects, involving core dynamics, plays a non-trivial rôle while the theory of defect motions involves rather the long term and somewhat mythical limit of an isolated defect in uniform motion [23]. 


\section{References}

[1] For a recent review see the communications concerning the transition to turbulence in the proceedings of the workshop, Common Trends in Particule and Condensed Matter Physics, Les Houches (March 1983) Physics Reports, to appear.

[2] Cross, M. C. and Newell, A. C., Convection patterns in large aspect ratio systems, Bell labs. preprint 1983.

[3] Newell, A. C. and Whitehead, J., J. Fluid Mech. 38 (1979) 279 ;

SEGEL, L. A., J. Fluid Mech. 38 (1969) 203.

[4] Siggia, E. and Zippelius, A., Phys. Rev. Lett. 47 (1981) 835.

[5] Cross, M. C., Phys. Rev. A 27 (1983) 490.

[6] Manneville, P. and Piquemal, J. M., Phys. Rev. A 28 (1983) 1774.

[7] Cross, M. C., Phys. Rev. A 25 (1982) 1065.

[8] a) Greenside, H. S., Coughran, W. M., Jr and Schryer, N. L., Phys. Rev. Lett. 49 (1982) 726.

b) Greenside, H. S. and Coughran, W. M., Jr, Non-linear Pattern Formation near the Threshold of Rayleigh-Bénard Convection, Bell labs. preprint 1983.

[9] Manneville, P., J. Physique 44 (1983) 563.

[10] Swift, J. and Hohenberg, P., Phys. Rev. A 15 (1977) 319 (Appendix A).

[11] Pomeau, Y. and Manneville, P., J. Physique Lett. 40 (1979) L-609.

[12] ZIPPElius, A. and SiggiA, E. D., Stability of finite amplitude convection, Preprint NSF-ITP-82-42.

[13] Manneville, P., J. Physique 44 (1983) 759.

[14] Normand, C., Z. Angew. Math. Phys. 32 (1981) 81.

[15] Richtmyer, R. D. and Morton, K. W., Difference Methods for Initial Value Problems (Wiley, New York) 1967.

[16] Acton, F. S., Numerical Methods that Work (Harper and Row, New York) 1970.

[17] Ahlers, G. and Behringer, R. P., Phys. Rev. Lett. 40 (1978) 712.

Maurer, J. and LibChaber, A., J. Physique Lett. 41 (1980) L-515.

[18] Gollub, J. P., McCarriar, A. R. and Steinman, J. F., J. Fluid Mech. 125 (1982) 259.

[19] The effects of the coupling between the dislocation and the secondary flow has been considered by E. Dubois-Violette, E. Guazzelli and J. Prost : Philos. Mag. A in press.

[20] Bergé, P., private communication. See also reference 18.

[21] Busse, F. H. and Clever, R. M., J. Fluid Mech. 91 (1979) 319.

[22] Manneville, P. and Pomeau, Y., Physica 1D (1980) 219.

[23] Pomeau, Y., Zaleski, S. and Manneville, P., Phys. Rev. A 27 (1983) 2710. Appendix C. 